\title{
In vivo assessment of endothelial permeability of coronary lesions with variable degree of stenosis using an albumin-binding MR probe
}

\author{
Leif-Christopher Engel 1,2,3 . Ulf Landmesser ${ }^{2,3} \cdot$ Youssef S. Abdelwahed ${ }^{2} \cdot$ Kevin Gigengack $^{2} \cdot$ Thomas Wurster $^{2}$. \\ Costantia Manes ${ }^{2}$. Carsten Skurk ${ }^{2}$. Alexander Lauten ${ }^{2}$. Andreas Schuster ${ }^{4,5} \cdot$ Michel Noutsias $^{6} \cdot$ Bernd Hamm $^{8}$. \\ Rene M. Botnar ${ }^{7,10} \cdot$ Boris Bigalke $^{2} \cdot$ Marcus R. Makowski ${ }^{8,9}$
}

Received: 14 January 2021 / Accepted: 17 May 2021 / Published online: 10 July 2021

(c) The Author(s) 2021

\begin{abstract}
MR imaging with an albumin-binding probe enables the visualization of endothelial permeability and damage in the arterial system. The goal of this study was to compare signal enhancement of lesions with different grades of stenosis segments on molecular CMR in combination with the albumin-binding probe gadofosveset. This prospective clinical study included patients with symptoms suggestive of coronary artery disease (CAD). Patients underwent gadofosveset-enhanced cardiovascular magnetic resonance (CMR) imaging and x-ray angiography (QCA) within $24 \mathrm{~h}$. CMR imaging was performed prior to and $24 \mathrm{~h}$ following the administration of gadofosveset. Contrast-to-noise ratios (CNRs) between segments with different grades of stenosis were compared. Overall, $n=203$ segments of 26 patients were included. Lesions with more than $>70 \%$ stenosis demonstrated significantly higher CNRs compared to lesions $<70 \%$ ( $7.6 \pm 8.3$ vs. $2.5 \pm 4.9 ; p<0.001)$. Post-stenotic segments of lesions $>70 \%$ stenosis showed significant higher signal enhancement compared to segments located upstream of these lesions ( $7.3 \pm 8.8$ vs. $2.8 \pm 2.2 ; p=0.02)$. No difference in signal enhancement between segments proximal and distal of lesions with stenosis greater than $50 \%$ was measured ( $3.3 \pm 2.8$ vs. $2.4 \pm 2.7 ; p=0.18$ ). ROC analysis for the detection of lesions $\geq 70 \%$ revealed an area under the curve of 0.774 (95\% CI $0.681-0.866$ ). This study suggests that relevant coronary stenosis and their down-stream segments are associated with increased signal enhancement on Gadofosveset-enhanced CMR, suggesting a higher endothelial permeability in these lesions. An albumin-binding MR probe could represent a novel in vivo biomarker for the identification and characterization of these vulnerable coronary segments.
\end{abstract}

Keywords Endothelial damage $\cdot$ Stenosis $\cdot$ Molecular MRI $\cdot$ QCA $\cdot$ Target-specific MR probe

Leif-Christopher Engel, Ulf Landmesser, Boris Bigalke and Marcus R. Makowski contributed equally

Leif-Christopher Engel

leifengel@hotmail.com

1 Department of Cardiology, German Heart Center, Munich, Germany

2 Department of Cardiology, Charité Campus Benjamin Franklin, Universitätsmedizin Berlin, Berlin, Germany

3 Berlin Institute of Health (BIH), Berlin, Germany

4 Department of Cardiology and Pulmonology, German Centre for Cardiovascular Research (DZHKPartner Site), Göttingen, Germany

5 Department of Cardiology, Royal North Shore Hospital, The Kolling Institute, Northern Clinical School, University of Sydney, 5th Floor, Acute Services Building, Reserve Road, St Leonard's, Sydney, NSW, Australia
6 Mid-German Heart Center, Department of Internal Medicine III (KIM-III), Division of Cardiology, Angiology and Intensive Medical Care, University Hospital Halle-Wittenberg, Halle (Saale), Germany

7 Escuela de Ingeniería, Pontificia Universidad Católica de Chile, Santiago, Chile

8 Department of Radiology, Charité - Universitätsmedizin Berlin, Berlin, Germany

9 Department of Radiology, Klinikum Rechts Der Isar, TU München, München, Germany

10 Division of Imaging Sciences and Biomedical Engineering, King's College London, London, UK 


$\begin{array}{ll}\text { Abbreviations } \\ \text { AUC } & \text { Area under the curve } \\ \text { CAD } & \text { Coronary artery disease } \\ \text { CMR } & \text { Cardiovascular magnetic resonance imaging } \\ \text { CNR } & \text { Contrast-to-noise ratio } \\ \text { GE-CMR } & \begin{array}{l}\text { Gadofosveset-enhanced cardiovascular mag- } \\ \text { netic resonance imaging }\end{array} \\ \text { ICA } & \text { Invasive coronary angiography } \\ \text { MLD } & \text { Minimal lumen diameter } \\ \text { MR } & \text { Magnetic resonance imaging } \\ \text { NSTEMI } & \text { Non-ST-elevation myocardial infarction } \\ \text { QCA } & \text { Quantitative coronary angiography }\end{array}$

\section{Introduction}

Endothelial permeability and damage precedes the manifestation of atherosclerosis and plays a major role in its development [1]. The measurement of flow-mediated vasodilatation of the brachial artery is the noninvasive gold standard to evaluate endothelial function [2]. However, it was shown that this technique correlates only moderately with other vascular beds including the coronaries [3]. Molecular cardiac magnetic resonance (CMR) imaging using targeted molecular probes enables the visualization of biological processes, which cannot be detected by morphological imaging approaches such as cardiac computed tomography CT [4, 5]. The albumin-binding magnetic resonance-probe (MR-probe) gadofosveset-trisodium was shown to have properties comparable to the Evan's blue dye, which represents a surrogate marker for endothelial permeability in the arterial system [4, 5]. Currently, there is only limited data on the association between local endothelial damage or dysfunction and the degree of stenosis [6].

The goal of this study was to compare signal enhancement of lesions with different grades of stenosis segments on molecular CMR. We hypothesize that lesions with higher grade of luminal narrowing are associated with stronger signal enhancement after application of an albumin-binding MR probe, which is considered as a surrogate parameter for endothelial permeability."

\section{Methods}

\section{Study population}

This study included subjects with indication for invasive catheterization based on their symptoms of coronary artery disease (CAD), such as stable chest pain and acute coronary syndrome (unstable angina; Non-ST-elevation myocardial infarction/NSTEMI). All subjects were prospectively recruited between April 2015 and June 2016. All patients underwent CMR-imaging prior to (native scan) and 24-h following the administration of gadofosveset-trisodium (gadofosveset-enhanced-scan;GE-CMR). Subsequently, invasive catheterization and quantitative coronary angiography (QCA) was performed in each patient. Exclusion criteria were hemodynamically unstable patients (cardiogenic shock, rising cardiac enzymes or malignant arrhythmias, ST-elevation-myocardial-infarction;STEMI), pregnancy, patients with renal insufficiency (creatinine clearance $<30 \mathrm{ml} / \mathrm{min}$ ), a history of coronary stenting, or who were not able to give their written consent (i.e. $<18$ years of age, mental disorders) and common contraindication to CMR imaging (i.e. allergy to gadolinium-based contrast agents, claustrophobia, metallic - items such as cochlear implants, central nervous system aneurysm clips, pacemakers/defibrillators). All patients gave written informed consent and the study was approved by the ethics committee of Charite Universitätsmedizin Berlin for clinical investigations (EA4/112/14) and performed in accordance with the Declaration of Helsinki.

\section{Cardiac magnetic resonance imaging}

All subjects underwent a CMR exam in a 3-Tesla-scanner (Magnetom Skyra, Siemens-Healthcare,Erlangen,Germany) using an 18-channel matrix coil. In all patients, CMR imaging was performed twice: Natively and $24 \mathrm{~h}$ following the administration of gadofosveset-trisodium $(0.03 \mathrm{mmol} /$ $\mathrm{kg} /$ body-weight), which was administered intravenously through a catheter in an antecubital vein. In this study, we used a post-contrast imaging time of around 24-h to reduce the signal from gadofosveset in the coronary lumen and to get optimal wall-to-lumen-contrast as demonstrated previously [5].

Continuous monitoring of vital signs throughout the entire CMR scan was performed with a 4-lead ECG. Patients with elevated cardiac enzymes were monitored using a CMR-compatible blood pressure monitor and blood oxygenation sensorAfter the acquisition of scout scans to identify the major structures of the heart, a cine 4-chamber-view was used to determine trigger delay and acquisition window, followed by a TI scout to determine the patient-specific inversion time to null signal from blood. For whole heart MR coronary angiography a T2-prep prepared FLASH (fast low angle shot) sequence was used including the following imaging parameters: field of view $340 \times 340 \mathrm{~mm}$; acquisition matrix $256 \times 256$; reconstruction matrix $512 \times 512$; acquisition slice thickness $1.3 \mathrm{~mm}$; acquisition slice number $80-100$; reconstruction spatial resolution $0.65 \times 0.65 \times 0.65 \mathrm{~mm}$; repetition time/echo time $3.5 \mathrm{~ms} / 1.42 \mathrm{~ms}$; flip angle 20 degrees. An inversion recovery (IR) prepared 3-dimensional (3D) T1W turbo FLASH (fast low angle shot) sequence with fat suppression (FatSat) was used for whole heart coronary vessel wall imaging. 
Electrocardiogram - triggering and a navigator-gated free breathing technique in coronal orientation was part of each acquistion. To null blood using a region of interest to determine the most accurate value, we adjusted the patientspecific inversion time (range $270 \mathrm{~ms}$ to $300 \mathrm{~ms}$ ) using the TI scout sequence. The following acquisition parameters were included: inversion time $250 \pm 15 \mathrm{~ms}$; field of view $340 \times 340 \mathrm{~mm}$; acquisition matrix $256 \times 256$; reconstruction matrix $512 \times 512$; acquisition slice thickness $1.3 \mathrm{~mm}$; acquisition slice number $80-100$; reconstruction spatial resolution $0.65 \times 0.65 \times 0.65 \mathrm{~mm}$; repetition time/echo time $4.1 \mathrm{~ms} / 1.3 \mathrm{~ms}$; flip angle 15 degrees. The navigator gating window width was $1.5-2.5 \mathrm{~mm}$. Depending on the patient's heart rate, the data acquisition window duration time varied from $84 \mathrm{~ms}$ to $120 \mathrm{~ms}$ depending. The trigger delay and acquisition window were adjusted according to the phase with minimal motion of the right coronary artery (RCA) as determined by cine MR imaging.

\section{Invasive catheterization and Quantitative Coronary Angiography (QCA)}

Two perpendicular projections were acquired for all coronary arteries and angiographic view with minimal foreshortening were chosen as described previously [7]. Pixel size was determined with automated distance calibration and all analyses were performed on frames demonstrating optimal luminal opacification. All QCA measurements were performed using a semi-automated edge detection system (QAngio XA 7.3,Medis,Leiden,Netherlands). The proximal and distal limits of the lesion were set manually before a semi-automated edge-detection software then delineated the lesion contours and provided the reference vessel diameter and luminal diameter at maximal obstruction. The minimal lumen diameter (MLD) was defined as the smallest luminal diameter in the segment of interest. The diameter stenosis in $\%$ was automatically calculated. Lesions were stratified into five different categories based on the extent of stenosis: $100 \%, 70-99 \%, 50-69 \%, 30-49 \%, 0-29 \%$.

\section{CMR image analysis}

CMR analysis, was performed using a dedicated image analysis software (Osirix-3.6.1,Geneva,Switzerland). Signal enhancement of coronary segments was determined on GECMR and the precontrast CMR-scan according to a 9-Segment model. Contrast-to-noise ratio (CNR) was defined as the difference in signal enhancement between the coronary segment and blood divided by the background noise (SI lesion-SI blood/noise). The standard deviation of the signal enhancement in a region of interest ventrally to the patient's chest was used to obtain the background noise [5].
Table 1 Baseline patients' characteristics and medical treatment upon admission

\begin{tabular}{ll}
\hline & All patients $(n=26)$ \\
\hline Age, $\mathrm{y}$ & $69.2 \pm 13.2$ \\
Male, $n(\%)$ & $17(65.4)$ \\
Weight, $\mathrm{kg}$ & $81.2 \pm 19.6$ \\
BMI, $\mathrm{kg} / \mathrm{m} 2$ & $27.4 \pm 6.8$ \\
Risk factors & \\
Hypercholesterolemia, $n(\%)$ & $13 / 26(50)$ \\
Hypertension, $n(\%)$ & $22 / 26(84.6)$ \\
Diabetes mellitus, $n(\%)$ & $8 / 26(30.8)$ \\
Smoking, $n(\%)$ & $12 / 26(46.2)$ \\
Family history of CAD, $n(\%)$ & $5 / 26(19.2)$ \\
Laboratory findings & \\
Troponin T, ng/ml & $141.2 \pm 380.4$ \\
CK, UI/l & $147.3 \pm 104.3$ \\
CK-MB, UI/l & $29.4 \pm 30.9$ \\
Creatinine, mg/dl & $1.0 \pm 0.3$ \\
C-reactive protein, mg/dl & $36.9 \pm 76.2$ \\
Platelets, $\times 109$ & $252.2 \pm 56.5$ \\
Total cholesterol, mg/dl & $182.4 \pm 46.8$ \\
Triglyceride, mg/dl & $160.2 \pm 103.7$ \\
HDL cholesterol, mg/dl & $46.3 \pm 14.3$ \\
LDL cholesterol, mg/dl & $113.1 \pm 42.9$ \\
Medication & \\
Aspirin & $16 / 26(61.5)$ \\
Statin & $9 / 26(34.6)$ \\
Beta-blocker & $11 / 26(42.3)$ \\
ACEI and/or ARB & $16 / 26(61.5)$ \\
\hline
\end{tabular}

\section{Statistics}

We used the SPSS-software (IBM-SPSS-Statistics-Version24) for statistical analysis. Continuous variables were reported as mean standard deviation or median with interquartile range (25th and 75 th percentiles). Nominal variables were reported as percentage or frequencies. Differences in continuous variables were calculated using the Kruskal-Wallis-test or analysis-of-variance (ANOVA), when appropriate. Nominal variables were compared among the three groups using chi-square and Fisher-exact-tests, when appropriate. A 2-tailed $p$-value $<0.05$ was reported as statistically significant.

\section{Results}

Clinical baseline characteristics $(n=26)$ are shown in the supplemental Table 1. Coronary segments, which were not correctly visible on invasive catheterization due to overlap of other vessels of foreshortening (i.e. wrong projections), were 

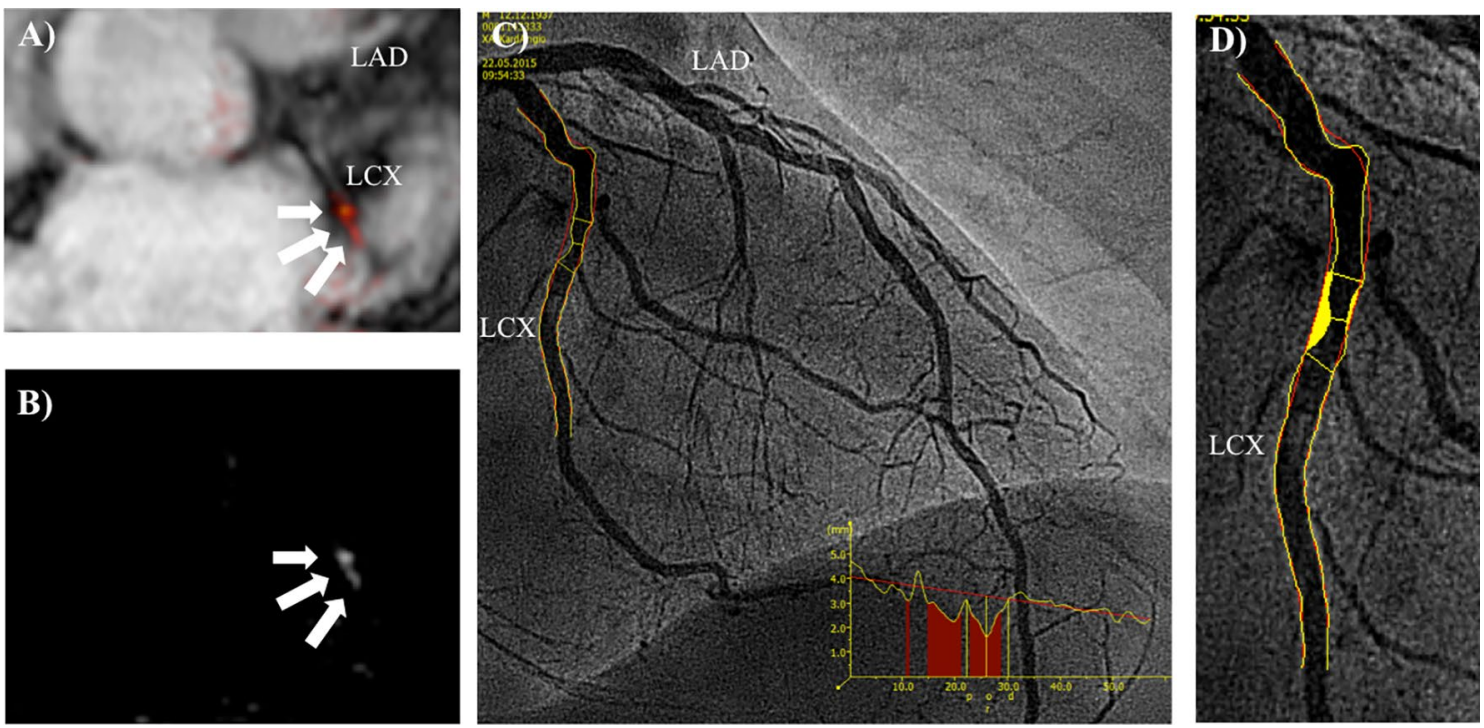

Fig. 1 Representative images following the administration of an albumin-binding MR probe for the assessment of endothelial permeability and damage at sites of significant stenosis. To highlight the anatomical relationship between the uptake of the albumin-binding-MR probe (b) and morphology from the MR angiography, images were fused in a way comparable to positron emission tomography/computed tomography (a). Subsequent Invasive catheterization demonstrated a stenosis in the left circumflex artery (LCX) (c). The assessment using quantitative coronary angiography (QCA) revealed a stenosis of $73 \%$ (d). This sample case illustrates that CMR in combination with an albumin-binding probe is able to clearly identify coronary artery stenosis greater than $70 \%$. Signal enhancement following application of gadofosveset-trisodium is known to be a surrogate for endothelial permeability and damage, which may be increased in severe stenosis
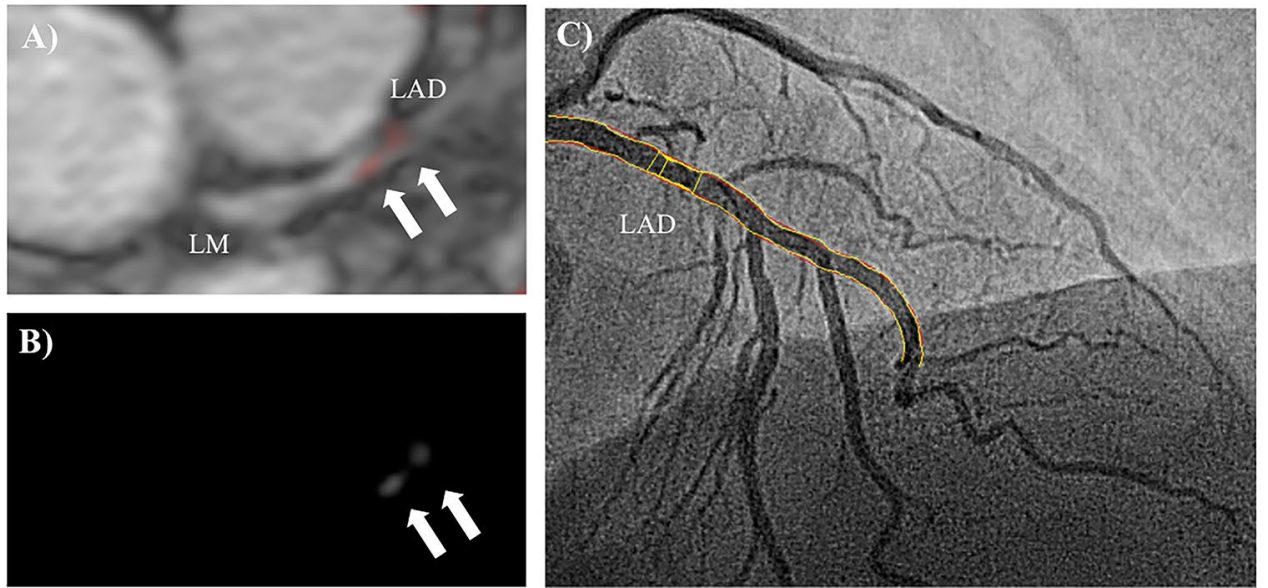

Fig. 2 Representative images demonstrating on minimal signal enhancement at sites of non-relevant stenosis following the administration of an albumin-binding MR probe. To highlight the anatomical relationship between the uptake of the albumin-binding-MR probe

excluded. In addition, $n=31$ segments, mainly in the distal left arterial desceding artery (LAD), left circumflex artery (LCX) or right coronary artery (RCA), had to be excluded due to insufficient image quality on CMR. Consequently, 203/234 coronary segments were available for analysis in the coronary artery scan $24 \mathrm{~h}$ following the administration of the albumin-binding-MR-probe. (b) and morphology from the MR angiography, images were fused in a way comparable to positron emission tomography/computed tomography (a). Subsequent Invasive catheterization demonstrated only a minimal stenosis in the left arterial descending artery (LAD) (C)

\section{Invasive catheterization and quantitative coronary angiography (QCA)}

The total amount of coronary segments containing a total occlusion were $n=8$. The number of coronary segments with a stenosis of $70-99 \%, 50-69 \%, 30-49 \%$ and $0-29 \%$ were $n=13, n=31, n=83$ and $n=68$, respectively. The mean length of lesions in the stenosis-category $70-99 \%, 50-69 \%$, 
Fig. 3 a ROC analysis for the detection of stenosis $\geq 50 \%$ and $\geq 70 \%$ revealed an AUC of 0.667 (95\% CI 0.586-0.748) and 0.774 (95\% CI $0.681-$ $0.866)$ respectively. Differences between $\mathbf{b}$ prestenotic, intrastenotic (50-69\% stenosis) and poststenotic segments and $\mathbf{c}$ prestenotic, intrastenotic $(\geq 70 \%$ stenosis) and poststenotic segments are demonstrated

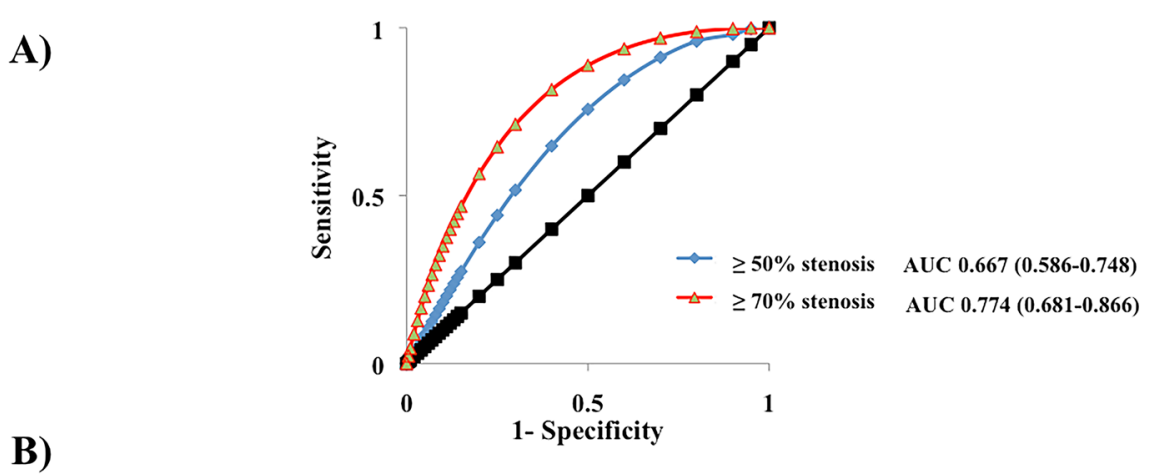

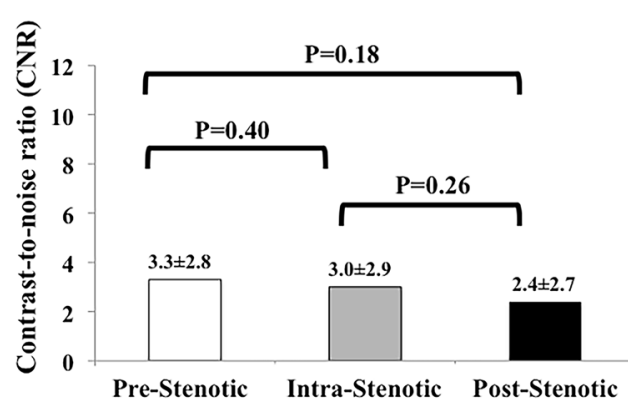

$50-69 \%$ Stenosis

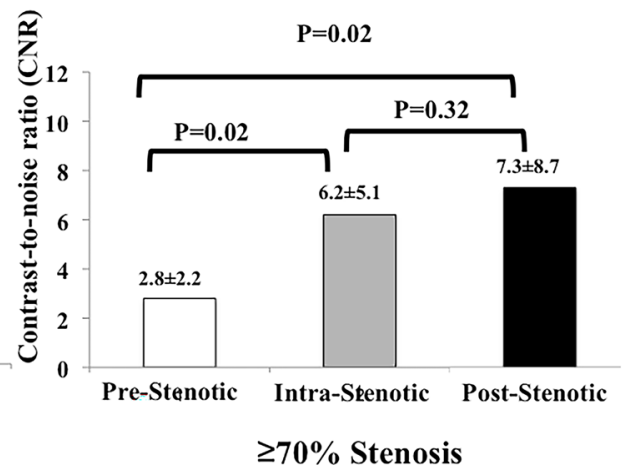

$30-49 \%$ and $0-29 \%$ was $7.9 \pm 4.4 \mathrm{~mm}, 6.4 \pm 2.7 \mathrm{~mm}$, $5.0 \pm 3.8 \mathrm{~mm}$ and $3.8 \pm 3.5 \mathrm{~mm}$, respectively.

\section{Comparison of signal enhancement between segments with variable degree of stenosis on Gadofosveset-enhanced CMR}

Lesions greater than $70 \%$ stenosis demonstrated significantly higher contrast-to-noise ratio (CNR) values as compared to lesions below that threshold $(7.6 \pm 8.31$ vs. $2.5 \pm 4$ $.9 ; \mathrm{p}<0.001)$. Figure 1 demonstrates a sample where high signal enhancement at sites of lesions with greater than $70 \%$ stenosis is visible (see Fig. 1), whereas Fig. 2 shows only mild signal enhancement on Gadofosveset-enhanced CMR at sites of lesion with only minimal to mild stenosis (see Fig. 2). ROC analysis for the detection of lesions greater than $50 \%$ and greater than $70 \%$ revealed an area under the curve (AUC) of 0.667 (95\% CI 0.586-0.748) and 0.774 (95\% CI 0.681-0.866), respectively (see Fig. 3A). There was no difference in CNR values between segments located proximally and distally of lesions with stenosis greater than $50 \%(3.3 \pm 2.8$ vs. $2.4 \pm 2.7 ; p=0.18)$ (see Fig. 3B). However, segments located distally of lesions with greater than $70 \%$ stenosis showed significant higher signal enhancement compared to segments located proximally of these lesions ( $7.3 \pm 8.8$ vs. $2.8 \pm 2.2 ; p=0.02)$ (see Fig. 3C).

\section{Comparison of signal enhancement between the pre-contrast and Gadofosveset-enhanced CMR exam}

Significant differences regarding signal enhancement between the non-contrast-enhanced baseline scan and the gadofosveset-enhanced scan (GE-CMR) were seen in all segments with a stenosis $\geq 50 \%(4.7 \pm 6.1 \mathrm{vs} .3 .0 \pm 3.9 ; p=0.03)$. No differences in signal enhancement between the non-contrast-enhanced baseline scan and the gadofosveset-enhanced scan (GE-CMR) were observed in all segments containing a stenosis $<50 \%(2.5 \pm 5.3$ vs. $2.5 \pm 6.3 ; p=0.487)$. Similarily, signal enhancement between the non-contrast-enhanced baseline scan and the gadofosveset-enhanced scan (GECMR) differed significantly between segments with a stenosis $\geq 70 \%$ ( $7.6 \pm 8.3$ vs. $1.9 \pm 3.4 ; p=0.003$ ). In contrast, segments containing a stenosis $<70 \%(2.5 \pm 4.9$ vs. $2.7 \pm 6.0$; $p=0.407$ ) demonstrated equal signal enhancement between the non-contrast-enhanced baseline scan and the gadofosveset-enhanced scan (GE-CMR).

\section{Discussion}

This study suggests that severe stenotic lesions are associated with an increase in endothelial permeability and damage. Lesions causing luminal narrowing impair endothelial function, especially in post-stenotic segments. 


\section{Stenosis severity and Vulnerable Plaque}

The measurement of brachial artery flow-mediated vasodilatation is the noninvasive gold standard to determine endothelial function and represents a surrogate parameter for predicting cardiovascular disease progression and outcome [2].

Local and low-grade systemic inflammation promotes the development of atherosclerosis. Finally, the Canakinumab Anti-inflammatory Thrombosis Outcomes Study (CANTOS) has provided strong evidence in support of the inflammation hypothesis [8]. In this regard, it was shown recently that the C-reactive protein to albumin ratio (CAR), which is seen as a novel marker of inflammation, had a very strong diagnostic value in detecting significant CAD [9].

MR-imaging with an albumin-binding probe instead yields information on the presence and extent of endothelial permeability and damage rather than inflammation, which plays a also major role in the development of atherosclerosis and plaque formation [1]. Additionally, it has been suggested that signal enhancement after application of an albumin-binding MR probe is a surrogate marker for the detection of high-risk coronary plaques [4]. Until recently it was widely believed that these plaques were only associated with mainly mild to moderate luminal narrowing [7]. However a study suggested later on that it is twice as likely for a lesion to be a thin-cap fibroatheroma in severe stenosis that in a non-severe stenosis [10], supporting our assumption that signal enhancement after application of an albumin-binding MR-probe is a not only a surrogate marker for the detection of vulnerable plaques but also of severe stenosis. For, plaque growth is associated with intraplaque hypoxemia leading to the proliferation of fragile neovessels with "leaky" endothelium [4, 11], which is the target of the albumin-binding MR-probe used in this study. Inward expansion in case of stenosis may have the same effect on neoangiogenesis as outward expansion of a plaque (i.e. positive remodeling) which is a feature of high-risk plaques [11]. The higher signal enhancement in post-stenotic segments suggests that the constantly reduced blood flow in poststenotic segments results in endothelial damage, reflected by increased endothelial permeability. In line with this, Heinen et al. observed a decrease in vasodilate function as assessed by flow-mediated vasodilation down stream of significant stenosis [6].

\section{Clinical implication}

MR imaging with an albumin-binding probe has been shown to detect and visualize vascular and endothelial damage, rather than relevant areas of stenosis, which is commonly defined as $>70 \%$ stenosis in a major coronary vessel, or $>50 \%$ stenosis in combination with fractional flow reserve $\leq 0.8$ [12]. Based on our data, these sites may not only be characterized by increased endothelial permeability itself, but also may negatively affect the endothelium downstream, predisposing them for plaque formation. Therefore, the added information of MR imaging with an albumin-binding MR-probe could improve risk stratification beyond risk scores such as the SYNTAX score, for instance, which relies solely on coronary anatomy and lesion characteristics [13].

\section{Limitations}

Only 26 patients were included, limiting the findings in this study and preventing us to include a meaningful correlation analysis of CNR and stenosis. Findings of this small feasibility study were not compared to flow-mediated vasodilatation of the brachial artery. However, previous studies demonstrated that there is strong evidence that the albumin-leakage-sign in CMR represents a surrogate marker for pathological endothelial function and endothelial permeability [4,5]. Finally, MRimaging with an albumin-binding probe is still associated with a relatively long scan time for the assessment of the coronary enhancement in vivo, which currently limits its applicability in a wide clinical setting. Using more advanced motion correction techniques in combination with undersampled image reconstruction (e.g. compressed sensing), this limitation may be solved in the future [14].

\section{Conclusion}

This study suggests that high-grade stenotic coronary lesions are associated with an increase in endothelial permeability. Lesions causing luminal narrowing greater than $70 \%$ may impair endothelial function especially in post-stenotic segments. An albumin-binding MR probe could represent a novel in vivo-biomarker for identification and characterization of these vulnerable coronary segments.

Author contributions We confirm that the work is original and has not been published or is under consideration for publication in any other journal in whole or in part except as an abstract. All authors have significantly contributed to the manuscript: BB, UL, MRM. conceived the hypothesis and together with L.C.E. and C.M. conducted the statistical analyses and wrote the first draft of the manuscript. KG, TW, GG provided expert technical assistance. CS, DML, AL performed invasive catheterizations. QCA measurements were performed by YA and $\mathrm{KG}, \mathrm{AS}, \mathrm{BH}, \mathrm{MN}, \mathrm{RMB}$ provided critical input at all stages and cooperated for the study design, critically reviewed and contributed to the manuscript. All authors have read and approved the manuscript. For all authors, there are no conflicts of interest.

Funding Open Access funding enabled and organized by Projekt DEAL. 


\section{Declarations}

Conflict of interest Authors Leif-Christopher Engel, Ulf Landmesser, Youssef S. Abdelwahed, Kevin Gigengack, Costantina Manes, Thomas Wurster, Georg Girke, Carsten Skurk, David M. Leistner, Alexander Lauten, Andreas Schuster, Michel Noutsias, Bernd Hamm, Rene M. Botnar, Boris Bigalke, Marcus R. Makowski have declares that he has no conflict of interest.

Ethical approval All procedures performed in studies involving human participants were in accordance with the ethical standards of Charite Universitätsmedizin Berlin and/or national research committee and with the 1964 Helsinki declaration and its later amendments or comparable ethical standards. This article does not contain any studies with animals performed by any of the authors.

Informed consent Informed consent was obtained from all individual participants included in the study.

Open Access This article is licensed under a Creative Commons Attribution 4.0 International License, which permits use, sharing, adaptation, distribution and reproduction in any medium or format, as long as you give appropriate credit to the original author(s) and the source, provide a link to the Creative Commons licence, and indicate if changes were made. The images or other third party material in this article are included in the article's Creative Commons licence, unless indicated otherwise in a credit line to the material. If material is not included in the article's Creative Commons licence and your intended use is not permitted by statutory regulation or exceeds the permitted use, you will need to obtain permission directly from the copyright holder. To view a copy of this licence, visit http://creativecommons.org/licenses/by/4.0/.

\section{References}

1. Davignon J, Ganz P (2004) Role of endothelial dysfunction in atherosclerosis. Circulation. 109:III27-32

2. Ras RT, Streppel MT, Draijer R, Zock PL (2013) Flow-mediated dilation and cardiovascular risk prediction: a systematic review with meta-analysis. Int J Cardiol 168:344-351

3. Anderson TJ, Uehata A, Gerhard MD, Meredith IT, Knab S, Delegrange D, Lieberman EH, Ganz P, Creager A, Yeung AC, Selwyn AP (1995) Close relation of endothelial function in the human coronary and peripheral circulation. J Am Coll Cardiol 26:1235-1241

4. Phinikaridou A, Andia ME, Protti A, Indermuehle A, Shah A, Smith A, Warley A, Botnar RM (2012) Noninvasive magnetic resonance imaging evaluation of endothelial permeability in murine atherosclerosis using an albumin-binding contrast agent. Circulation 126:707-719

5. Lobbes MB, Heeneman S, Passos VL, Welten R, Kwee RM, van der Geest RJ, Wiethoff AJ, Caravan P, Misselwitz B, Daemen
MJ, van Engelshoven JM, Leiner T, Kooi ME (2010) Gadofosveset-enhanced magnetic resonance imaging of human carotid atherosclerotic plaques: a proof of-concept study. Invest Radiol 45:275-281

6. Heinen Y, Stegemann E, Sansone R, Benedens K, Wagstaff R, Balzer J, Rassaf T, Lauer T, Kelm M, Heiss C (2010) Local association between endothelial dysfunction and intimal hyperplasia: relevance in peripheral artery disease. J Am Heart Assoc 4:e001472. https://doi.org/10.1161/JAHA.114.001472

7. Shah PK (2003) Mechanisms of Plaque Vulnerability and Rupture. J Am Coll Cardiol 19(41):15S-22S

8. Ridker PM, Libby P, MacFadyen JG et al (2018) Modulation of the interleukin- 6 signalling pathway and incidence rates of atherosclerotic events and all-cause mortality: analyses from the Canakinumab Anti-Inflammatory Thrombosis Outcomes Study (CANTOS). Eur Heart J 39:3499-3507

9. Tanriverdi Z, Gungoren F, Tascanov MB, Besli F, Altiparmak IH (2020) Comparing the diagnostic value of the c-reactive protein to albumin ratio with other inflammatory markers in patients with stable angina pectoris. Angiology 71(4):360-365

10. Tian J, Dauerman H, Toma C, Samady H, Itoh T, Kuramitsu S, Domei T, Jia H, Vergallo R, Soeda T, Hu S, Minami Y, Lee H, Yu B, Jang IK (2014) Prevalence and characteristics of TCFA and degree of coronary artery stenosis: an OCT, IVUS, and angiographic study. J Am Coll Cardiol. 19(64):672-680

11. Virmani R, Burke AP, Farb A, Kolodgie FD (2006) Pathology of the vulnerable plaque. J Am Coll Cardiol 18:C13-C18

12. Neglia D, Rovai D, Caselli C, Pietila M, Teresinska A, AguadéBruix S, Pizzi MN, Todiere G, Gimelli A, Schroeder S, Drosch T, Poddighe R, Casolo G, Anagnostopoulos C, Pugliese F, Rouzet F, Le Guludec D, Cappelli F, Valente S, Gensini GF, Zawaideh C, Capitanio S, Sambuceti G, Marsico F, Perrone Filardi P, Fernández-Golfín C, Rincón LM, Graner FP, de Graaf MA, Fiechter M, Stehli J, Gaemperli O, Reyes E, Nkomo S, Mäki M, Lorenzoni V, Turchetti G, Carpeggiani C, Marinelli M, Puzzuoli S, Mangione M, Marcheschi P, Mariani F, Giannessi D, Nekolla S, Lombardi M, Sicari R, Scholte AJ, Zamorano JL, Kaufmann PA, Underwood SR, Knuuti J, EVINCI Study Investigators (2015) Detection of significant coronary artery disease by noninvasive anatomicaland functional imaging. Circ Cardiovasc Imaging. 8(3):e002179

13. Hayıroğlu Mİ, Keskin M, Uzun AO, Bozbeyoğlu E, Yıldırımtürk Ö, Kozan Ö (2018) Pehlivanoğlu S Predictive value of SYNTAX score II for clinical outcomes in cardiogenic shock underwent primary percutaneous coronary intervention; a pilot study. Int J Cardiovasc Imaging 34(3):329-336

14. Aitken AP, Henningsson M, Botnar RM, Schaeffter T, Prieto C (2015) 100\% Efficient threedimensional coronary MR angiography with two-dimensional beat-to-beat translational and bin-to-bin affine motion correction. Magn Reson Med 74:756-764

Publisher's Note Springer Nature remains neutral with regard to jurisdictional claims in published maps and institutional affiliations. 